EXTENDED REPORT

\title{
Association study of detoxification genes in age related macular degeneration
}

\author{
H Esfandiary, U Chakravarthy, C Patterson, I Young, A E Hughes
}

Br J Ophthalmol 2005;89:470-474. doi: 10.1136/bjo.2004.047340

See end of article for authors' affiliations

....................

Correspondence to: Anne Hughes, Centre for Vision Sciences, Queen's University Belfast, Institute of Clinical Science, Royal Victoria Hospital, Belfast BT126BA, UK; a.hughes@ qub.ac.uk

Accepted for publication 11 August 2004
Background/aims: Age related macular degeneration (AMD) is a complex disorder leading to loss of central vision, and identification of risk factors associated with susceptibility to AMD has been a key objective for ophthalmic genetics research for almost a decade. This association study has examined genetic polymorphisms in 12 candidate genes as possible risk factors for predisposition to the development of the exudative variant of AMD in a Northern Irish population. The choice of genes was based on their function in the breakdown of industrial pollutants, cigarette smoke, defence against oxidative stress, or involvement in the general ageing process.

Methods: Up to five single nucleotide polymorphisms (SNPs) were typed for CYP1A1, CYP1A2, CYP2E1, CYP2D6, EPHX1, MnSOD, AhR, NAT2, CAT, GPX1, PON1, and ADPRT1 by multiplex snapshot single base primer extension method. Genes showing high linkage disequilibrium (LD) between SNPs were analysed by haplotype analysis. Genes showing low LD were assessed using individual SNPs based on genotypes.

Results: After correction for number of genes/SNPs tested, no significant association with AMD was found although several genes merit further investigation. This study suggests that a coding SNP in EPHX1 (Y113H) may be important in AMD and supports a previous observation of an association with exudative AMD. In addition, haplotype analysis highlighted ADPRT1, CYP2D6, and AhR as worthy of further study. Conclusion: This study has identified a number of genes requiring further investigation including EPHX1, ADPRT1, CYP2D6, and AhR.
A ge related macular degeneration (AMD) is now recognised as the most common cause of acquired visual impairment in people over 60 years of age, and is estimated to affect up to 25 million individuals in the Western world. The problem will be compounded by demographic changes which suggest that the number of people aged over 60 will triple from some 600 million worldwide in 2000 to nearly two billion by 2050, with the United Kingdom predicted to have about 16 million people over the age of 60 by $2040 .^{1}$

The current understanding is that AMD represents a multifactorial disorder involving environmental and genetic factors. Familial aggregation, segregation, and twin studies have clearly established that genetic predisposition has a role in the aetiology of AMD. ${ }^{2}$ It is assumed that multiple genes are involved, although the number of those has not been reliably estimated nor the contribution of each to the disease. As in other complex traits, the extent of genetic heterogeneity cannot be estimated easily so that the influences of many genes and their interactions are difficult to ascertain even in individual families. A number of environmental risk factors have been shown to be associated with the disease. These include smoking, hypertension, diet, lifetime exposure to sunlight, sex, and iris colour. Thus, AMD can be considered to be a complex trait where genetic studies are confounded by its late onset, reduced penetrance, and genetic heterogeneity.

Considering that environmental effects may be under genetic control, we have investigated the role of polymorphisms in genes encoding enzymes involved in detoxification of reactive oxygen species that are produced from the breakdown of industrial pollutants including cigarette smoke. A number of genes involved in the first line of defence against oxidative stress such as catalase (CAT), manganese superoxide dismutase (MnSOD or SOD2), glutathione peroxidase 1 (GPXI), and paraoxonase 1 (PONl) as well as several cytochrome P450 gene superfamily members (CYP) have been typed for several common single nucleotide polymorphisms (SNPs) in a Northern Irish population with exudative $\mathrm{AMD}$ and a population based control sample.

\section{SUBJECTS AND METHODS \\ Sample recruitment}

Subjects with exudative AMD were attendees at the ophthalmology clinics at the Royal Victoria Hospital, Belfast, Northern Ireland, between 2001 and 2002. All subjects were 50 years of age or older and had a confirmed diagnosis of exudative AMD in at least one eye, which was based on angiographic assessment performed in the fluorescein angiogram reading centre at Queen's University Belfast. Whole blood from participants was obtained through venepuncture after informed consent was given. Control subjects were participants in the EUREYE study, a population based random selection of subjects over the age of 65 who were resident in Northern Ireland during the recruitment period (2001-2), and had provided informed consent for use of their DNA for studies on ageing and vision. Control subjects were selected on the basis of fundus grading which showed absence of any drusen or pigmentary irregularities. Ethical approval for this project was obtained from the local research ethics committee and the study adhered strictly to the tenets of the Declaration of Helsinki on the participation of human volunteers in clinical studies.

Abbreviations: AA, amino acid; ADPRT1, ADP-ribosyltransferase 1; $A h R$, aryl hydrocarbon receptor; $A M D$, age related macular degeneration; CAT, catalase; $C S N P s$, coding SNPs; EPHX1, epoxide hydrolase 1; GPX1, glutathione peroxidase 1; LD, linkage disequilibrium; MAF, minor allele frequency; $M n S O D$, manganese superoxide dismutase; NAT2, n-acetyl transferase 2; PCR, polymerase chain reaction; $\mathrm{PON1}$, paraoxonase 1; SNPs, single nucleotide polymorphisms; SOD2, superoxide dismutase 2 
Candidate genes, SNP selection, and validation

CYP1A1, CYP1A2, CYP2D6, CYP2E1, epoxide hydrolase 1 (EPHX1), SOD2, aryl hydrocarbon receptor (AhR), n-acetyl transferase 2 (NAT2), CAT, PON1, GPXl, and ADP-ribosyltransferase l (ADPRTl) were selected for investigation.

Coding SNPs (cSNPs) and non-cSNPs with minor allele frequency of $15 \%$ were amplified by polymerase chain reaction (PCR) and validated by sequencing, using standard methodology. Thereafter, multiplex PCR assays were designed for each gene to amplify up to four amplicons (Qiagen). Snapshot primer extension (ABI) was used for SNP genotyping.

\section{Statistical methods}

Having first checked Hardy-Weinberg equilibrium using the $\chi^{2}$ goodness of fit test, the $\chi^{2}$ test for contingency tables or Fisher's exact probability test was used to compare allele frequencies between cases and controls. These comparisons were conducted using a p value threshold of 0.001 to allow for the numbers of analyses considered. Haplotype frequencies were estimated using the EM algorithm as implemented in the Arlequin program. ${ }^{3}$ The resulting estimates in cases and controls were then compared using a likelihood ratio $\chi^{2}$ test statistic but with significance assessed empirically using the FastEHPlus software. ${ }^{4}$ An empirical $p$ value is obtained by comparing the observed test statistic to its permutation distribution, which was approximated by 10000 random permutations of the case/control labels. Haplotype comparisons were conducted using a p value threshold of 0.005 to allow for the number of analyses considered. The extent of linkage disequilibrium between pairs of SNPs was quantified using Lewontin's $\mathrm{D}^{\prime}$ value. ${ }^{5}$ For rare alleles our study was of sufficient size to have $80 \%$ power to detect a $15 \%$ difference in allele frequencies between cases and controls (for example, allele frequencies of $20 \%$ versus $5 \%$ ) as statistically significant using the reduced level of significance $(\mathrm{p}<0.001)$, which makes allowance for multiple comparisons. For more common alleles the study still had $80 \%$ power to detect a rather larger $20 \%$ difference (for example, allele frequencies of $40 \% v 20 \%$ ) using the same level of significance.

\section{RESULTS}

A total of 94 cases and 95 controls were analysed for all the genes, with the exception of ADPRT1 which numbered 162 cases and 188 controls. Average age of cases was 76.6 years and that of controls 78.8 years. Cases represent end stage exudative AMD and controls presented no abnormal macular features. Cases were $61.8 \%$ female $/ 38.2 \%$ male, whereas controls reflected equal numbers of males and females.

Twelve genes were analysed with up to five SNPs (40 SNPs/ 7520 genotypes; table 1). The frequencies of common haplotypes are shown for each gene in addition to $\mathrm{D}^{\prime}$ values between pairs of SNPs (table 2). The genes covered fell into two categories as depicted by the $\mathrm{D}^{\prime}$ measure. Most genes showed a high $\mathrm{D}^{\prime}$ reflecting strong LD between all SNPs

Table 1 Genes and SNPs assessed for AMD

\begin{tabular}{|c|c|c|c|c|c|c|c|}
\hline \multicolumn{3}{|l|}{ Gene } & \multicolumn{5}{|c|}{ SNP details } \\
\hline Gene & Size (kb) & Exons & Location & rs number & SNP & AA change & MAF \\
\hline \multirow[t]{4}{*}{ ADPRTI } & 47 & 23 & Intron 1 & 2048426 & $G>A$ & - & 0.216 \\
\hline & & & Exon 17 & 1136410 & $\mathrm{~T}>\mathrm{C}$ & V762A & 0.089 \\
\hline & & & Intron 17 & 2271349 & $G>T$ & - & 0.089 \\
\hline & & & Intron 20 & 747657 & $G>C$ & - & 0.216 \\
\hline \multirow[t]{4}{*}{ AhR } & 47 & 12 & Promotor & 765623 & $A>G$ & - & 0.274 \\
\hline & & & Intron 1 & 2237298 & $C>T$ & - & 0.447 \\
\hline & & & Intron 4 & 2158041 & $C>T$ & - & 0.289 \\
\hline & & & Exon 10 & 2066853 & $G>A$ & R554K & 0.089 \\
\hline \multirow[t]{4}{*}{ CAT } & 33 & 13 & Intron 1 & 480575 & $A>G$ & - & 0.279 \\
\hline & & & Intron 6 & 1408035 & $A>T$ & - & 0.200 \\
\hline & & & Exon 9 & 769217 & $C>T$ & D389D & 0.200 \\
\hline & & & Intron 9 & 2284367 & $\mathrm{~T}>\mathrm{C}$ & - & 0.200 \\
\hline \multirow[t]{5}{*}{ CYPIAl } & 6 & 7 & Promotor & 2470893 & $G>A$ & - & 0.453 \\
\hline & & & Intron 1 & 2606345 & $T>G$ & - & 0.271 \\
\hline & & & Exon 7 & 1799814 & $C>A$ & $\mathrm{~T} 461 \mathrm{~N}$ & 0.047 \\
\hline & & & Exon 7 & 1048943 & $A>G$ & $1462 \mathrm{~V}$ & 0.037 \\
\hline & & & $3^{\prime}$ UTR & 4646903 & $\mathrm{~T}>\mathrm{C}$ & - & 0.079 \\
\hline \multirow[t]{2}{*}{ GPX1 } & 1.4 & 2 & Promotor & 3448 & $G>A$ & - & 0.232 \\
\hline & & & Promotor & 3811699 & $A>G$ & - & 0.311 \\
\hline \multirow[t]{4}{*}{ NAT2 } & 9.9 & 2 & Intron 1 & 923796 & $C>G$ & - & 0.437 \\
\hline & & & Exon 2 & 1801280 & $\mathrm{~T}>\mathrm{C}$ & $1114 \mathrm{~T}$ & 0.484 \\
\hline & & & Exon 2 & 1799930 & $G>A$ & R197Q & 0.332 \\
\hline & & & Exon 2 & 1799931 & $A>G$ & K268R & 0.468 \\
\hline \multirow[t]{3}{*}{ SOD2 } & 14 & 5 & Exon 2 & 1799725 & $\mathrm{~T}>\mathrm{C}$ & V16A & 0.500 \\
\hline & & & Intron 4 & 2758330 & $A>G$ & - & 0.200 \\
\hline & & & 3' UTR & 1967802 & $\mathrm{~T}>\mathrm{C}$ & - & 0.232 \\
\hline CYPIA2 & 7.7 & 7 & Exon 7 & $\mathrm{~T} 1545 \mathrm{C}$ & $\mathrm{T}>\mathrm{C}$ & $\mathrm{N} 515 \mathrm{~N}$ & 0.247 \\
\hline \multirow[t]{2}{*}{ CYP2D6 } & 5 & 9 & Intron 2 & 2854740 & $C>T$ & - & 0.516 \\
\hline & & & Exon 9 & 2743456 & $C>T$ & Y489Y & 0.205 \\
\hline \multirow[t]{3}{*}{ CYP2E1 } & 11.7 & 9 & Promotor & 2070673 & $\mathrm{~T}>\mathrm{A}$ & - & 0.179 \\
\hline & & & Intron 5 & 2070675 & $C>T$ & - & 0.189 \\
\hline & & & Exon 8 & 2515641 & $C>T$ & $\mathrm{~F} 421 \mathrm{~F}$ & 0.153 \\
\hline \multirow[t]{4}{*}{ EPHXI } & 35.4 & 9 & Promotor & 2854447 & $C>T$ & - & 0.205 \\
\hline & & & Exon 1 & 1877724 & $C>T$ & - & 0.211 \\
\hline & & & Exon 3 & 1051740 & $\mathrm{~T}>\mathrm{C}$ & Y113H & 0.274 \\
\hline & & & Exon 4 & 2234922 & $A>G$ & H139R & 0.163 \\
\hline \multirow[t]{4}{*}{ PONI } & 26.8 & 9 & Intron 1 & 2237583 & $G>A$ & - & 0.305 \\
\hline & & & Exon 3 & 854560 & $A>G$ & L55M & 0.337 \\
\hline & & & Exon 6 & 662 & $G>A$ & Q192R & 0.300 \\
\hline & & & Exon 9 & 854552 & $A>G$ & - & 0.307 \\
\hline
\end{tabular}

$M A F$, minor allele frequency in control subjects $(n=95)$; $A A$, amino acid. 
typed, indicating that the entire gene was contained within a single haplotype block. For ADPRT1, SOD2, AhR, CAT, and NAT2, three common haplotypes accounted for over $90 \%$ of the possible haplotypes, which was further evidence for LD. These genes were assessed on the basis of common haplotype frequencies which improved the robustness of this association study. In all of these genes no significant differences in haplotype frequencies were found $(\mathrm{p}>0.05)$ (table 2$)$. The figures do not include a correction for the number of genes examined.

The second group of genes (CYP2El, PON1, and EPHXl) had low $\mathrm{D}^{\prime}$ values reflecting low LD between SNPs within each gene and indicated that more than one haplotype block was observed. For these genes assessment of haplotypes was inappropriate and hence analysis was based on comparison of individual SNPs (table 3). Three SNPs in the EPHXl gene showed $\mathrm{p}$ values below 0.05 , one of which was located in the coding region (Y113H). Genotype frequencies in the CYP2E1 and PONl were not significantly different in cases and controls.

\section{DISCUSSION}

Previously association studies have examined individual SNPs within candidate genes as risk factors for the development of AMD usually by assessing cSNPs for differences in allele frequencies. A major caveat with all these studies is their small size, and lack of replication makes conclusions less robust.

The aim of our study was to examine candidate genes likely to be important in the protection against oxidative stress which may be an important mechanism in the development of AMD. Initial haplotype analysis of the ADPRTl gene produced a significant finding $(\mathrm{p}=0.006)$ with one haplotype ("1111") being more common in the control group inferring a protective effect similar to that reported for the ACE gene. However, on replication in a separate group of cases and controls the trend was reversed $(p=0.186)$. The results presented for ADPRTl $(p=0.042)$ represent pooled data for both groups. This gene encodes a chromatin associated enzyme ADP-ribosyltransferase which is induced by the presence of DNA strand breaks. It has a role in DNA repair and the recovery of cells from DNA damage. A positive correlation has been found between ADPRTl and lifespan, with human cells displaying about five times the activity of rat cells. ${ }^{6}$ The function of ADPRT1 in maintaining genomic stability and its potential involvement in the ageing process was the basis for its selection as a candidate gene in the development of AMD, which is essentially a degenerative

Table 2 Haplotypes, frequencies, and LD for genes examined based on 94 cases and 95 controls

\begin{tabular}{|c|c|c|c|c|c|c|}
\hline \multirow[b]{2}{*}{ Gene } & \multirow[b]{2}{*}{ Size (kb) } & \multirow[b]{2}{*}{ Common haplotypes* } & \multicolumn{2}{|c|}{ Haplotype frequency } & \multirow{2}{*}{$\begin{array}{l}\text { Range of pairwise } \\
D^{\prime} \text { values }\end{array}$} & \multirow{2}{*}{$\begin{array}{l}\text { Haplotype } \\
\text { p Value }\end{array}$} \\
\hline & & & Controls & Cases & & \\
\hline \multirow[t]{3}{*}{ ADPRT1† } & 47 & 1111 & 0.726 & 0.654 & $0.97-1.0$ & 0.042 \\
\hline & & 2112 & 0.136 & 0.191 & & \\
\hline & & 2222 & 0.125 & 0.133 & & \\
\hline \multirow{3}{*}{ AhR } & 47 & 1111 & 0.535 & 0.633 & $0.88-1.0$ & 0.017 \\
\hline & & 2221 & 0.236 & 0.17 & & \\
\hline & & 1212 & 0.089 & 0.122 & & \\
\hline \multirow[t]{3}{*}{ CAT } & 33 & 1111 & 0.721 & 0.699 & $1.0-1.0$ & 0.68 \\
\hline & & 2222 & 0.200 & 0.231 & & \\
\hline & & 2111 & 0.079 & 0.065 & & \\
\hline \multirow{4}{*}{ CYPIAI } & 6 & 21111 & 0.452 & 0.36 & $0.95-1.0$ & 0.14 \\
\hline & & 11111 & 0.277 & 0.36 & & \\
\hline & & 12111 & 0.149 & 0.118 & & \\
\hline & & 12211 & 0.048 & 0.081 & & \\
\hline \multirow[t]{3}{*}{ GPX1 } & 1.4 & 11 & 0.458 & 0.468 & $0.99-1.0$ & 0.87 \\
\hline & & 12 & 0.311 & 0.324 & & \\
\hline & & 21 & 0.232 & 0.207 & & \\
\hline \multirow{3}{*}{ NAT2 } & 9.9 & 2212 & 0.431 & 0.335 & $0.99-1.0$ & 0.19 \\
\hline & & 1121 & 0.332 & 0.378 & & \\
\hline & & 1111 & 0.179 & 0.223 & & \\
\hline \multirow[t]{4}{*}{ SOD2 } & 14 & 211 & 0.494 & 0.468 & $0.97-1.0$ & 0.71 \\
\hline & & 112 & 0.232 & 0.229 & & \\
\hline & & 121 & 0.194 & 0.245 & & \\
\hline & & 111 & 0.074 & 0.059 & & \\
\hline \multirow[t]{3}{*}{ CYP2D6 } & 5 & 11 & 0.516 & 0.415 & $0.57-1.0$ & 0.064 \\
\hline & & 21 & 0.279 & 0.388 & & \\
\hline & & 22 & 0.205 & 0.197 & & \\
\hline \multirow{3}{*}{ CYP2E1 } & 11.7 & 111 & 0.678 & 0.685 & $0.05-0.70$ & $\mathrm{n} / \mathrm{a}$ \\
\hline & & 121 & 0.127 & 0.087 & & \\
\hline & & 212 & 0.116 & 0.054 & & \\
\hline \multirow[t]{6}{*}{ EPHXI } & 35.4 & 1111 & 0.525 & 0.372 & $0.02-1.0$ & $\mathrm{n} / \mathrm{a}$ \\
\hline & & 1121 & 0.116 & 0.186 & & \\
\hline & & 2221 & 0.103 & 0.149 & & \\
\hline & & 1112 & 0.094 & 0.103 & & \\
\hline & & 2211 & 0.088 & 0.113 & & \\
\hline & & 1122 & 0.055 & 0.036 & & \\
\hline \multirow[t]{8}{*}{ PONI } & 26.8 & 1211 & 0.282 & 0.197 & $0.13-1.0$ & $\mathrm{n} / \mathrm{a}$ \\
\hline & & 1122 & 0.164 & 0.131 & & \\
\hline & & 2111 & 0.159 & 0.18 & & \\
\hline & & 1112 & 0.115 & 0.091 & & \\
\hline & & 1121 & 0.079 & 0.071 & & \\
\hline & & 2121 & 0.07 & 0.084 & & \\
\hline & & 1111 & 0.068 & 0.137 & & \\
\hline & & 2211 & 0.035 & 0.092 & & \\
\hline
\end{tabular}




\begin{tabular}{|c|c|c|c|c|c|}
\hline \multirow[b]{2}{*}{ Gene } & \multirow[b]{2}{*}{ SNP rs number } & \multirow[b]{2}{*}{ Allele } & \multicolumn{2}{|c|}{ Allele frequency } & \multirow[b]{2}{*}{ p Value } \\
\hline & & & Controls & Cases & \\
\hline CYP1A2 & $\mathrm{T} 1545 \mathrm{C}$ & $\begin{array}{l}1 \text { (T) } \\
2 \text { (C) }\end{array}$ & $\begin{array}{l}0.75 \\
0.25\end{array}$ & $\begin{array}{l}0.69 \\
0.31\end{array}$ & 0.15 \\
\hline \multirow[t]{6}{*}{ CYP2E1 } & 2070673 & $1(\mathrm{~T})$ & 0.82 & 0.86 & 0.28 \\
\hline & & 2 (A) & 0.18 & 0.14 & \\
\hline & 2070675 & 1 (C) & 0.81 & 0.80 & 0.86 \\
\hline & & $2(\mathrm{~T})$ & 0.19 & 0.20 & \\
\hline & 2515641 & 1 (C) & 0.85 & 0.81 & 0.32 \\
\hline & & $2(\mathrm{~T})$ & 0.15 & 0.19 & \\
\hline \multirow{8}{*}{ EPHXI } & 2854447 & 1 (C) & 0.79 & 0.70 & 0.029 \\
\hline & & $2(\mathrm{~T})$ & 0.21 & 0.30 & \\
\hline & 1877724 & 1 (C) & 0.79 & 0.70 & 0.039 \\
\hline & & $2(\mathrm{~T})$ & 0.21 & 0.30 & \\
\hline & 1051740 & 1 (T) & 0.73 & 0.61 & 0.013 \\
\hline & & 2 (C) & 0.27 & 0.39 & \\
\hline & 2234922 & $1(\mathrm{~A})$ & 0.84 & 0.82 & 0.42 \\
\hline & & $2(G)$ & 0.16 & 0.18 & \\
\hline \multirow[t]{8}{*}{ PON1 } & 2237583 & 1 (G) & 0.69 & 0.65 & 0.36 \\
\hline & & $2(A)$ & 0.31 & 0.35 & \\
\hline & 854560 & 1 (T) & 0.66 & 0.68 & 0.77 \\
\hline & & $2(A)$ & 0.34 & 0.32 & \\
\hline & 662 & 1 (A) & 0.70 & 0.74 & 0.43 \\
\hline & & $2(G)$ & 0.30 & 0.26 & \\
\hline & 854552 & 1 (A) & 0.69 & 0.76 & 0.20 \\
\hline & & $2(G)$ & 0.31 & 0.24 & \\
\hline
\end{tabular}

disorder associated with ageing. ADPRTl retains some interest as an AMD susceptibility factor, but our results clearly illustrate the importance of replication of data. Initial positive associations in small studies need to be viewed with caution.

Haplotype analysis suggests that AhR and CYP2D6 may also warrant further investigation. The effect of many cytochromes is mediated by aryl hydrocarbon receptor (AhR) which is a ligand activated transcription factor involved in the regulation of biological responses to planar aromatic hydrocarbons. The protein product of CYP2D6 localises to the endoplasmic reticulum and is known to metabolise as many as $20 \%$ of commonly prescribed drugs.

Significant uncorrected $\mathrm{p}$ values were shown for three SNPs in the EPHXl gene, one of which is a cSNP (Y113H). $\mathrm{Y} 113 \mathrm{H}$ was shown to decrease in vitro activity of epoxide hydrolase by $40 \%^{7}$ and also to be weakly associated with wet AMD in a Japanese population. ${ }^{8}$ The tyrosine allele frequency in their patients was significantly higher than controls with an odds ratio of 1.43 and a p value of 0.034 . This is in contrast with our findings of a lower tyrosine allele frequency in cases $(p=0.013)$. Our $D^{\prime}$ values between SNPs suggest that EPHXI is contained in more than one haplotype block. Increasing the number of SNPs typed (together with larger sample sizes) would reveal the block structure and enable a more robust haplotype analysis to determine whether this gene is an important genetic determinant of AMD.

CYPIAl and CYP1A2 have both been implicated in other studies. ${ }^{9-11}$ The most strongly associated variants were not detected in our population, and we found no association with either gene suggesting that neither is a major susceptibility factor for AMD

Superoxide dismutase 2 (SOD2) or manganese superoxide dismutase (MnSOD) gene encodes an intramitochondrial free radical scavenging enzyme, which is the first line of defence against superoxide produced as a byproduct of oxidative phosphorylation. A cSNP (V16A) in this gene has been shown to be associated with wet AMD in a Japanese study. ${ }^{12}$ We assessed the same cSNP and two other intronic SNPs but were unable to demonstrate significant differences between cases and control $(\mathrm{p}=0.707)$. We therefore contend that SOD2 is unlikely to play a part in development of exudative AMD.

Paraoxonase is a $\mathrm{Ca}^{2+}$ dependent glycol protein that is associated with high density lipoprotein, and polymorphisms in this gene have been reported to be potential risk factors for wet AMD ${ }^{13}$ A positive association between wet AMD and two coding SNPs (L55M and Q192R) was observed in conjunction with increased levels of plasma oxidised low density lipoprotein levels. The potential significance of these SNPs in the pathogenesis of diabetic retinopathy in insulin dependent diabetes mellitus has also been investigated and similar results obtained for the L55M polymorphism. Owing to the low $\mathrm{D}^{\prime}$ value between SNPs, this gene was analysed on a genotype basis with the above mentioned cSNPs as well as two additional intronic SNPs. In contrast with previously published data, no significance was observed for any of the four SNPs and therefore larger studies using a more comprehensive subset of SNPs will be necessary to determine the true role, if any, of PONI in AMD pathogenesis.

In conclusion, our study has assessed a number of important candidate genes that have either been previously reported to be associated specifically with wet AMD or could potentially have a role in the pathogenesis of this degenerative disorder. Assessment of genes has been based either on haplotype analysis or on several individual SNPs of genes composed of more than one haplotype block. Larger numbers of genotypes have been carried out per gene than in earlier studies, improving confidence in observed results. With the aid of the multiplexing snapshot genotyping platform many SNPs have been assessed in over 180 cases and controls. While our AMD cohort is larger than many previously reported in similar association studies, we recognise that it lacks sufficient power for definitive identification of minor susceptibility factors. We can however support one previous association study in relation to the EPHXl gene while being unable to support others such as PON1. We also have evidence to suggest that ADPRTl, CYP2D6, and AhR may have a minor role in AMD susceptibility, and can recommend them for further study. We have determined the haplotype 
structure for several genes which may facilitate other studies by allowing haplotype tagged analysis of fewer SNPs.

In light of the large number of candidate genes which need to be assessed for their involvement in the pathogenesis of $\mathrm{AMD}$, the emergence of high throughput genotyping platforms such as microchip or bead array technology is anticipated to have a positive impact on the output quality of association studies in the future. This will surely revolutionise the study of complex genetic disease and lead to identification of major causative mutations or polymorphisms in many common diseases.

\section{Authors' affiliations}

H Esfandiary, U Chakravarthy, A E Hughes, Centre for Vision Sciences, Queen's University, Belfast, UK

C Patterson, I Young, Centre for Epidemiology and Population Studies, Queen's University, Belfast, UK

\section{REFERENCES}

1 National Statistics Office. National population projections. www.statistics.gov.uk/CCl/nugget.asp (accessed 9 Feb 2004).

2 Klaver CCW, Allikmets R. Genetics of macular dystrophies and implications for age-related macular degeneration. Dev Ophthalmol 2003;37:155-69.
3 Schneider S, Roessli D, Excofier L. Arlequin: a software for population genetics data analysis. Version 2.000. Geneva: Genetics and Biometry Laboratory, Department of Anthropology, University of Geneva, 2000

4 Zhao JH, Sham PC. Faster allelic association analysis using unrelated subjects. Hum Hered 2002:53:36-41.

5 Devlin B, Risch N. A comparison of linkage disequilibrium measures for finescale mapping. Genomics 1995;29:311-22.

6 Grube K, Burkle A. Poly(ADP-ribose) polymerase activity in mononuclear leukocytes of 13 mammalian species correlates with species-specific life span. Proc Natl Acad Sci USA 1992;89:11759-63.

7 Hassett C, Aicher L, Sidhu JS, et al. Human microsomal epoxide hydrolase: genetic polymorphism and functional expression in vitro of amino acid variants. Hum Molec Genet 1994;3:421-8.

8 Kimura K, Isashiki Y, Sonoda S, et al. Genetic association of manganese superoxide dismutase with exudative age-related macular degeneration. Am J Ophthalmol 2000;130:769-73.

9 Kawajiri K, Eguchi H, Nakachi K, et al. Association of CYPIA1 germ line polymorphisms with mutations of the p53 gene in lung cancer. Cancer Res 1996;56:72-6.

10 Nakachi K, Imai K, Hayashi S, et al. Genetic susceptibility to squamous cell carcinoma of the lung in relation to cigarette smoking dose. Cancer Res 1991;51:5177-80.

11 Xu X, Kelsey KT, Wiencke JK, et al. Cyłochrome P450 CYP1A1 polymorphism and lung cancer susceptibility. Cancer Epidemiol Biomarkers Prev 1996;5:687-92.

12 Ikeda T, Obayashi $\mathrm{H}$, Hasegawa G, et al. Paraoxonase gene polymorphisms and plasma oxidised low-density lipoprotein level as possible risk factors for exudative age-related macular degeneration. Am J Ophthalmol 2001;132:191-5

13 Kao Y-L, Donaghue K, Chan A, et al. A variant of paraoxonase (PON1) gene is associated with diabetic retinopathy in IDDM. J Clin Endocr Metab 1998:83:2589-92. 